\title{
Implications of heterostructural alloying for enhanced piezoelectric performance of $(\mathrm{Al}, \mathrm{Sc}) \mathrm{N}$
}

\author{
Kevin R. Talley, ${ }^{1,2}$ Samantha L. Millican, ${ }^{3}$ John Mangum, ${ }^{1}$ Sebastian Siol,,${ }^{2, *}$ Charles B. Musgrave, ${ }^{3}$ Brian Gorman, ${ }^{1}$ \\ Aaron M. Holder, ${ }^{2,3}$ Andriy Zakutayev, ${ }^{1,2}$ and Geoff L. Brennecka ${ }^{1, \dagger}$ \\ ${ }^{1}$ Colorado School of Mines, Metallurgical and Materials Engineering, Golden, Colorado 80401, USA \\ ${ }^{2}$ National Renewable Energy Laboratory, United States Department of Energy, Golden, Colorado 80401, USA \\ ${ }^{3}$ University of Colorado, Department of Chemical and Biological Engineering, Boulder, Colorado 80309, USA
}

(Received 21 November 2017; revised manuscript received 23 February 2018; published 29 June 2018)

\begin{abstract}
An understanding of the heterostructural implications on alloying in the aluminum nitride-scandium nitride $\operatorname{system}\left(\mathrm{Al}_{1-x} \mathrm{Sc}_{x} \mathrm{~N}\right)$ can highlight opportunities and design principles for enhancing desired material properties by leveraging nonequilibrium states. The fundamental thermodynamics, and therefore composition- and structuredependent mechanisms, underlying property evolution in this system have not been fully described, despite significant recent efforts driven by interest in enhanced piezoelectric performance. Practical realization of these enhanced properties, however, is hindered by the strong driving thermodynamic driving force for phase separation in the system, highlighting the need for increased study into the role of heterostructural alloying on the thermodynamics and composition-structure-property relationships in this system. With this need in mind, $a b$ initio computed alloy thermodynamics and properties are compared to combinatorial thin-film synthesis and characterization to develop a more complete picture of the structure and property evolution across the $\mathrm{Al}_{1-x} \mathrm{Sc}_{x} \mathrm{~N}$ composition space. The combination of structural frustration and a flattened free-energy landscape lead to substantial increases in electromechanical response. The energy scale of alloy metastability is found to be much larger than previously reported, helping to explain difficulties in achieving homogeneous materials with high scandium concentration. Scandium substitution for aluminum softens the wurtzite crystal lattice, and energetic proximity to the competing hexagonal boron-nitride structure enhances the piezoelectric stress coefficient. Overall, this work provides insight into the understanding of the structure-processing-property relationships in the $\mathrm{Al}_{1-x} \mathrm{Sc}_{x} \mathrm{~N}$ system, suggests material design strategies for even greater property enhancements, and demonstrates the increased property tunability and underexplored nature of nonequilibrium heterostructural alloys.
\end{abstract}

DOI: 10.1103/PhysRevMaterials.2.063802

\section{INTRODUCTION}

Alloys of materials having different crystal structures in their pure forms (heterostructural alloys) provide enhanced opportunities for accessing nonequilibrium states and otherwise inaccessible properties [1]. The $\mathrm{Al}_{1-x} \mathrm{Sc}_{x} \mathrm{~N}$ system is an interesting case study in heterostructural alloying as a tool for property improvement. This system, which combines wurtzitestructured $\mathrm{AlN}$ and rocksalt-structured $\mathrm{ScN}$, has been studied for decades, but the current work frames the system in terms of a heterostructural alloy, allowing us to focus on the underlying materials science and associated insights towards the design and discovery of new materials. The first report of $\mathrm{Al}_{1-x} \mathrm{Sc}_{x} \mathrm{~N}$ alloys showed no measurable solubility or intermediate compounds between wurtzite $\mathrm{AlN}$ and rocksalt $\mathrm{ScN}$, despite the presence of several stable intermetallic species in $\mathrm{N}$-deficient

\footnotetext{
*Present address: EMPA-Swiss Federal Laboratories for Materials Science and Technology, Überlandstrasse 129, 8600 Dübendorf, Switzerland.

†'geoff.brennecka@mines.edu

Published by the American Physical Society under the terms of the Creative Commons Attribution 4.0 International license. Further distribution of this work must maintain attribution to the author(s) and the published article's title, journal citation, and DOI.
}

regions of the phase diagram [2]. First-principles calculations later predicted scandium-nitride polymorph energies and suggested a metastable wurtzite phase that is $350 \mathrm{meV}$ per formula unit higher than the ground-state rocksalt phase [3]. Subsequent studies took one of two directions: epitaxial stabilization of the rocksalt structure for $x>0.5$ with interest in the electronic structure [4] or effects of alloying on piezoelectric properties of the wurtzite phase for $x<0.5$ [5]. After increases in the longitudinal piezoelectric strain coefficient $\left(d_{33}\right)$ were reported by Akiyama et al., the majority of the reported efforts focused on the effect of deposition parameters on crystal quality [6-10], residual stress [11,12], dielectric properties $[6,7,13]$, and overall electromechanical response $[11,14,15]$. Motivated by industrial interest in the improved performance in AlN-based piezoelectric microelectromechanical systems (piezoMEMS) devices, the breadth of this work was focused on compositions with $0<x<0.4$, where single-phase textured wurtzite materials could be synthesized. Recent work has focused on incorporation of these alloys into devices such as energy harvesters [16], ultrasonic transducers [17], and primarily in acoustic resonators [18-25], enabling alternative operation modes [26-28]. From a deployment perspective, the continued work on processing conditions highlights the difficulty of achieving high-quality materials with $x>0.2$, where misoriented grains degrade the macroscopic piezoelectric response [29-32]. In parallel with the experimental work, the theory 
community progressed from the previously mentioned firstprinciples polymorph energies [3] to first-principles mixing energies [4,33], lattice calculations [34], property calculations [35-37], and the effects of cation ordering on performance [38]. These calculations provided valuable insights but covered only limited ranges of $x$ with few crystal structures considered, which we show in this work did not sufficiently account for the role of heterostructural effects on the thermodynamics of these alloys and the resulting consequences on structure and properties.

Despite an enormous volume of research from academic and industrial laboratories in the past decade, many questions remain about the properties and associated fundamental mechanisms of $\mathrm{Al}_{1-x} \mathrm{Sc}_{x} \mathrm{~N}$ films across the entire composition range. Here the authors report a heterostructural alloy phase diagram and comprehensive view of the structure-processingproperty relationships across this thin-film system, and present this system as a case study in the value of heterostructural alloying for increased control and tunability of properties by accessing nonequilibrium states.

Density functional theory (DFT) calculations allow investigation into the origins of piezoelectric strain coefficient enhancement while complementary combinatorial synthesis enables the rapid experimental exploration of compositional space for structure, phase, optical, electrical, and mechanical properties for comparison to DFT results. The $\mathrm{Al}_{1-x} \mathrm{Sc}_{x} \mathrm{~N}$ system serves as an excellent example of the opportunity for property enhancement available through nonequilibrium states in heterostructural alloys that are accessible via energetic fabrication processes. This study provides a theorydriven understanding of the fundamental materials science concepts underlying this behavior in addition to experimental realization of predicted property enhancements. Additionally, it provides an opportunity to revisit the results of earlier studies on $\mathrm{Al}_{1-x} \mathrm{Sc}_{x} \mathrm{~N}$ as well as other $\mathrm{Al}_{1-x} R_{x} \mathrm{~N}$ such as $R=$ vanadium [39], tantalum [39,40], erbium [41], or chromium [42] in a different context, providing fundamental insights to guide future studies of these and other heterostructural alloy systems.

\section{METHODS}

High-throughput experimentation (HTE) in combination with $a b$ initio computation represents a robust tool set for screening and understanding materials systems and has found success in many fields, including transparent contacts for solar-cell applications [43] as well as interface analysis [44] and thermoelectrics [45]. These methods are uniquely capable of quickly screening compositional and/or process spaces as guides to materials selection, development, understanding, and process optimization [46]. The very features that enable HTE also limit the perfection that can be achieved for the specific region of material representing each data point. Combining HTE with computation, however, provides insight into the energy scale of alloy stability, assists with interpretation of microstructures and measured properties, and informs materials design by providing a quantitative measure of the instability of metastable structures and feasible deviations from equilibrium for practical applications.

\section{A. Computation}

$A b$ initio calculations were performed to computationally model the alloys. Mixing enthalpies were calculated using 72atom supercells with random cation configurations generated using the special quasirandom structures method [47] for each composition and phase. Alloy interaction parameters $(\Omega)$ for each polymorph were determined by fitting $\Delta H_{m}(x)$ using a second-order polynomial. The free energy of mixing $\Delta G_{m}$, which was used to construct the $T(x)$ phase diagram, was calculated as $\Delta G_{m}(x)=\Delta H_{m}(x)-T \Delta S_{m}(x)$, where $\Delta S_{m}(x)$ is the configuration entropy of the alloy in the regular solution model and given by $\Delta S_{m}(x)=-k_{b}[x \ln x+(1-x) \ln (1-x)]$. The miscibility gap (binodal line) was obtained from the common tangent construction in $\Delta G_{m}(x, t)$, while the spinodal gap was found from the condition $\delta^{2} \Delta G(x) / \delta x^{2}=0$. Four $\mathrm{Al}_{1-x} \mathrm{Sc}_{x} \mathrm{~N}$ polymorphs were considered for this study; wurtzite (WZ), hexagonal boron nitride (HBN), zinc blende (ZB), and rocksalt (RS), as shown in Fig. 1. The mixing
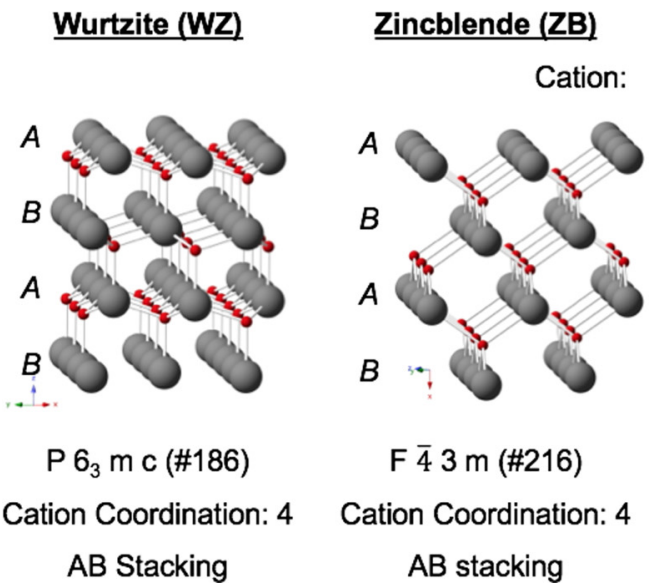

Hexagonal BN (HBN)

- Anion:

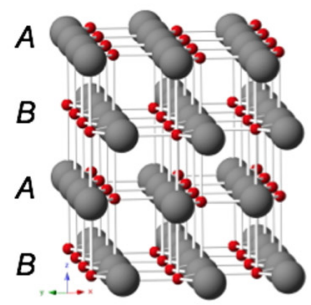

$P 6_{3} \mathrm{~m} \mathrm{c}$ (\#186)

Cation Coordination: 5 $A B$ stacking

\section{Rocksalt (RS)}

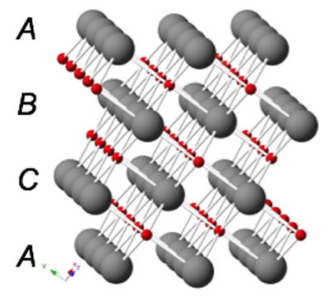

$\mathrm{F} m \overline{3} \mathrm{~m}(\# 225)$

Cation Coordination: 6

ABC Stacking

FIG. 1. Polymorphs of the $\mathrm{Al}_{x} \mathrm{Sc}_{1-x} \mathrm{~N}$ alloy system computed (all) and observed (left and right only) in this work; shown as ball-and-stick arrays of anions and cations to highlight the specific symmetry, coordination, and stacking arrangements. 
enthalpies $\Delta H_{m}$ were computed for each of these four structures across the entire composition range.

DFT calculations were performed within the Vienna $\mathrm{Ab}$ initio Simulation Package [48] (VASP) using the projector augmented-wave method [49]. Calculations were conducted at 19 compositions across the phase space for each crystal structure. Calculations utilized a $\Gamma$-centered $2 \times 2 \times 2$ $k$-point mesh with an energy cutoff of $620 \mathrm{eV}$. Geometry relaxations were conducted using the conjugate gradient algorithm and converged to within $10^{-7} \mathrm{eV}$ with an electronic self-consistency (SC) loop converged within $10^{-8} \mathrm{eV}$. For calculations of $\mathrm{Al}_{x} \mathrm{Sc}_{1-x} \mathrm{~N}$ alloy energetics, the PerdewBurke-Ernzerhof (PBE) generalized gradient approximation exchange-correlation functional [50] was used with an approximate van der Waals correction using the D2 method of Grimme [51]. An accurate description of the energy differences between polymorphs is necessary to correctly predict the heterostructural alloy phase diagram and identify critical compositions where structural transitions would be expected to occur. The van der Waals corrected method was chosen by benchmarking to accurate total energies of the $\mathrm{AlN}$ and $\mathrm{ScN}$ polymorphs computed using the random phase approximation within the adiabatic-connection fluctuation-dissipation theorem (RPAACFDT) [52]. The computed end-member polymorph energies using $\mathrm{PBE}+\mathrm{D} 2$ were found to reproduce the polymorph energy differences computed using the RPA to within $49 \mathrm{meV} /$ atom magnetocrystalline anisotropy energy (MAE) across all endmember polymorphs. Without the van der Waals correction, the PBE computed polymorph energy differences are $90 \mathrm{meV} /$ atom MAE, justifying the use of this correction (see SI for validation of the computational results using the optPBE van der Waals functional [53]). The elastic coefficients $\left(C_{i j k l}\right)$ and piezoelectric stress tensors $\left(e_{i j}\right)$ were evaluated from phonon and dielectric response calculations performed using finite differences and density functional perturbation theory [54], respectively. For comparison to experimental results, the piezoelectric strain tensors $\left(d_{i j}\right)$ are computed using the tensor relation $\left(e_{i j}=\sum_{k, l} d_{i j} C_{i j k l}\right)$.

\section{B. Experiment}

HTE comprises high-throughput synthesis, characterization, and analysis. As described below, the combinatorial methods employed here include simultaneous synthesis of compositionally graded thin films atop substrates of either uniform or graded temperatures as well as spatially resolved screening across the graded films.

Thin-film combinatorial sample libraries were produced by radio-frequency (rf) reactive cosputtering from two-inch-diameter elemental aluminum and scandium metallic targets, each $>99.99 \%$ pure. Additionally, a nitrogen cracker introduced a reactive nitrogen plasma to the deposition environment. Oxygen contamination was minimized by use of a cryogenic shroud surrounding the local deposition region. A radiative-heated substrate platen regulated the substrate temperature. For samples deposited on substrates with low thermal conductivity (Corning EagleXG), one quarter of the substrate was clamped to the platen, and the remaining three quarters hung in free space, creating a substrate temperature gradient in a direction perpendicular

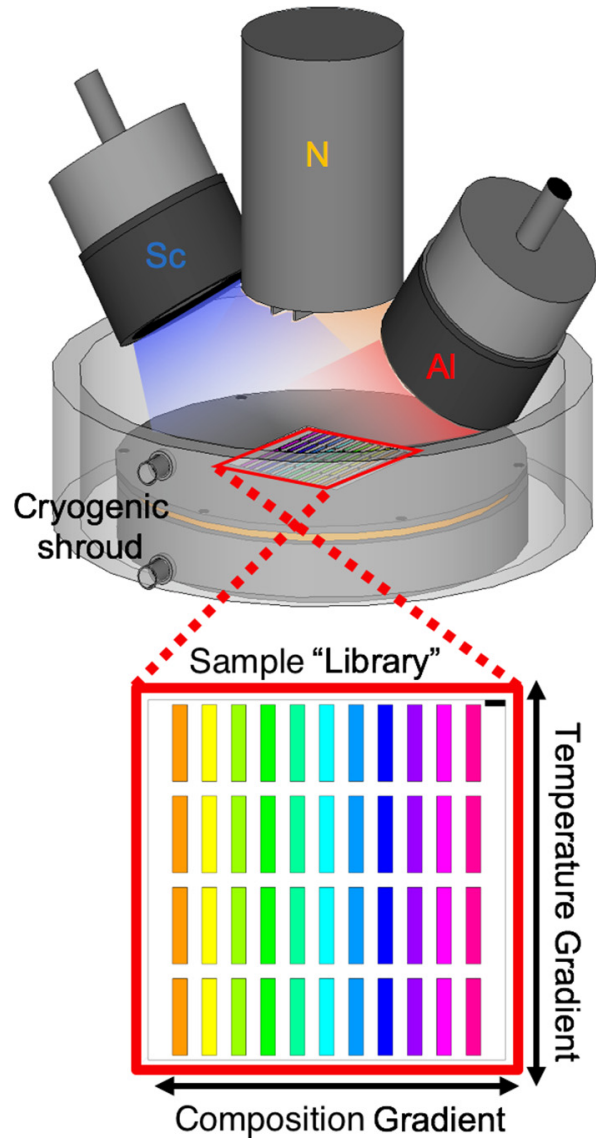

FIG. 2. Configuration of the combinatorial film deposition chamber used in this study. Shown are the three plasma sources, sample heater, cryogenic shroud, and resulting material library. The library is a $25 \mathrm{~cm}^{2}$ square that is binned into four rows and 11 columns, each capable of possessing a composition gradient and/or gradient in substrate deposition temperature.

to the composition gradient. Libraries deposited on $p$-type silicon were heated uniformly. A schematic of the deposition chamber and resulting combinatorial library is shown in Fig. 2. The libraries were subdivided into four rows and 11 columns in which the composition varied across columns and the substrate deposition temperature varied across rows. More information about the deposition chamber used in these experiments can be found in prior publications [55].

The processing conditions that were modified among the individual libraries are shown in Table I and include the sputtering power delivered to each target, substrate temperature, and substrate material. For all libraries, the vacuum base pressure was $10^{-7}$ torr, the processing pressure was $3 \times 10^{-3}$ torr, and the processing gas consisted of $6 \mathrm{sccm}$ argon in addition to a $350 \mathrm{~W}$ nitrogen plasma flowing at $4 \mathrm{sccm}$.

$\mathrm{X}$-ray diffraction was used to characterize the structures and textures of the thin films deposited by combinatorial methods. The diffraction pattern of each point was collected using a Bruker D8 Discovery diffractometer with a two-dimensional $(2 \theta$ and $\chi)$ area detector and collimated $\mathrm{Cu}_{K \alpha}$ radiation. The intensity counts were integrated across the detector in different dimensions, resulting in an intensity vs $2 \theta$ pattern for $\chi$ integration across the detector range and an intensity 
TABLE I. Processing parameters for growth of $\mathrm{Al}_{x} \mathrm{Sc}_{1-x} \mathrm{~N}$ thinfilm libraries. Some parameters remained constant during all depositions, while others varied among libraries.

\begin{tabular}{|c|c|c|c|c|}
\hline $\begin{array}{l}\text { Library } \\
\text { No. }\end{array}$ & $\begin{array}{l}\text { Power to } \\
\text { Al target } \\
\left(\mathrm{W} / \mathrm{cm}^{2}\right)\end{array}$ & $\begin{array}{l}\text { Power to } \\
\text { Sc target } \\
\left(\mathrm{W} / \mathrm{cm}^{2}\right)\end{array}$ & $\begin{array}{c}\text { Substrate } \\
\text { temperature } \\
\left({ }^{\circ} \mathrm{C}\right)\end{array}$ & $\begin{array}{c}\text { Substrate } \\
\text { type }\end{array}$ \\
\hline 1 & 4.6 & 0 & 410 & $p \operatorname{Si}(100)$ \\
\hline 2 & 0 & 4.6 & 410 & $p \operatorname{Si}(100)$ \\
\hline 3 & 4.6 & 1.2 & 410 & $p \operatorname{Si}(100)$ \\
\hline 4 & 4.6 & 2.3 & 410 & $p \operatorname{Si}(100)$ \\
\hline 5 & 4.6 & 3.5 & 410 & $p \operatorname{Si}(100)$ \\
\hline 6 & 4.6 & 4.6 & 410 & $p \operatorname{Si}(100)$ \\
\hline 7 & 4.6 & 3.5 & $100-200$ & EagleXG $\mathrm{SiO}_{2}$ \\
\hline 8 & 4.6 & 3.5 & $350-650$ & EagleXG $\mathrm{SiO}_{2}$ \\
\hline 9 & 4.6 & 4.6 & $100-200$ & EagleXG $\mathrm{SiO}_{2}$ \\
\hline 10 & 4.6 & 4.6 & $350-650$ & EagleXG $\mathrm{SiO}_{2}$ \\
\hline
\end{tabular}

vs $\chi$ pattern for $2 \theta$ integration in the range associated with a specific reflection [wurtzite (002) in this case]. For the case of $\mathrm{SiO}_{2}$ substrates, a background subtraction was used to remove the broad amorphous hump from the pattern. Each of the $2 \theta$ patterns was fit with a linear combination of Gaussian peaks to analyze the fraction of total diffracted intensity resulting from the wurtzite and rocksalt phases; this technique is often referred to as the disappearing-phase method [46]. An illustration of this method can be found in the Supplemental Material [56]. Each of the $\chi$ patterns was fit with a Gaussian peak to determine the full width at half maximum (FWHM) of the wurtzite (002) peak, producing a metric for the quality of the $c$-axis texture in the films which is equivalent to a typical $\omega$ rocking curve. The composition of each point on the material libraries was determined by x-ray fluorescence (XRF) using a Fischerscope X-ray XUV 773 vacuum tool and analysis software with calibration by end-member compositions of known thicknesses.

Transmission electron microscopy (TEM) micrographs were acquired with an FEI Talos F200X transmission electron microscope with scanning capabilities operating at an accelerating voltage of $200 \mathrm{keV}$. Specimens for TEM were prepared from deposited films via in situ focused ion-beam lift-out methods [57] using an FEI Helios Nanolab 600i SEM/FIB DualBeam workstation. Specimens were ion milled at $2 \mathrm{keV}$ and $77 \mathrm{pA}$ to remove $\mathrm{Ga}$ ion-beam damage and achieve a final thickness of approximately $80 \mathrm{~nm}$. Structural characterization was conducted by acquiring selected area electron-diffraction (SAED) patterns on an FEI Ceta 16M pixel CMOS camera at

TABLE II. Heater set point and resulting substrate temperature for the two substrates used in this study.

\begin{tabular}{|c|c|c|c|c|}
\hline $\begin{array}{l}\text { Substrate } \\
\text { type }\end{array}$ & $\begin{array}{l}\text { Heater set } \\
\text { point }\left({ }^{\circ} \mathrm{C}\right)\end{array}$ & $\begin{array}{l}\text { Hot } \\
\left({ }^{\circ} \mathrm{C}\right)\end{array}$ & $\begin{array}{l}\text { Middle } \\
\left({ }^{\circ} \mathrm{C}\right)\end{array}$ & $\begin{array}{l}\text { Cold } \\
\left({ }^{\circ} \mathrm{C}\right)\end{array}$ \\
\hline EagleXG SiO 2 & 150 & 150 & 130 & 100 \\
\hline EagleXG $\mathrm{SiO}_{2}$ & 350 & 400 & 345 & 265 \\
\hline EagleXG $\mathrm{SiO}_{2}$ & 420 & 450 & 391 & 305 \\
\hline$p \operatorname{Si}(100)$ & 286 & 410 & 410 & 410 \\
\hline
\end{tabular}

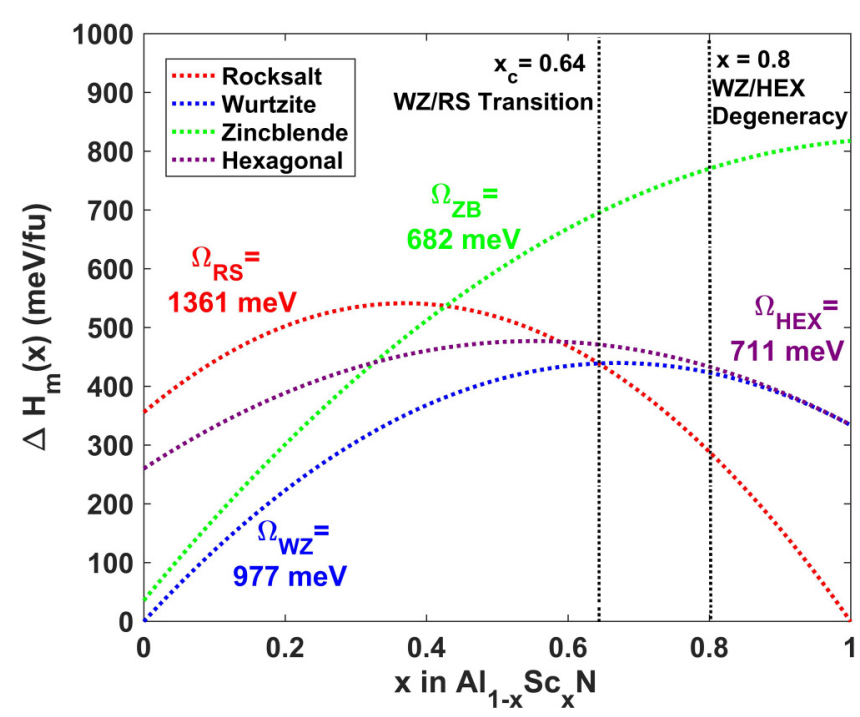

FIG. 3. Calculated mixing enthalpy $\left[\Delta H_{m}(x)\right]$ for $\mathrm{Al}_{1-x} \mathrm{Sc}_{x} \mathrm{~N}$ alloy polymorphs considered in this study.

a camera length of $410 \mathrm{~mm}$. Platinum from the FIB was used to calibrate the camera constant, allowing SAED reflections to be accurately measured and indexed. Chemical mapping was performed in the TEM using the Super-X energy-dispersive $\mathrm{X}$-ray spectroscopy (EDS) system equipped with four windowless silicon drift detectors, allowing for high count rates and chemical sensitivity (down to $0.5-1.0$ atomic percent).

\section{RESULTS}

\section{A. Computation}

Thermochemical calculations of the alloy end members and mixing enthalpies (shown in Fig. 3) predict that wurtzite is the ground-state structure for $\mathrm{AIN}$ and Al-rich $\mathrm{Al}_{1-x} \mathrm{Sc}_{x} \mathrm{~N}$, while rocksalt is the ground-state structure for $\mathrm{ScN}$ and $\mathrm{ScN}$-rich materials with a critical composition at $x=0.64$ (see Fig. 3). This is different from the critical composition reported by Zhang et al. of $x=0.56$, where the van der Waals correction was not employed and energy crossover points were incorrectly identified. This agrees with the results found by Höglund et al. [33]. Near this phase transition, the HBN structure-which is related to the wurtzite structure through a decrease in the $c / a$ ratio and increase in the internal $u$ parameter-is found to be very close in energy to that of the wurtzite and rocksalt structures and becomes degenerate at a composition of $x=$ 0.80 . This is reflected in the calculated lattice parameters of the wurtzite structure shifting towards the lattice parameters of the HBN structure near this composition (see Fig. 8 and associated discussion).

The $T(x)$ phase diagram shown in Fig. 4 is constructed from the free energy of mixing given by $\Delta G_{m}(x)=\Delta H_{m}(x)-$ $T \Delta S_{m}(x)$. We note that for the reported alloy phase diagram, the state-of-matter phase transitions from the solid state that will occur within the plotted temperature range are not included in this evaluation. However, the scale of metastability determined using this regular solution model remains correct and the single-phase alloys achieved at experimentally accessible temperatures require highly nonequilibrium conditions. The 


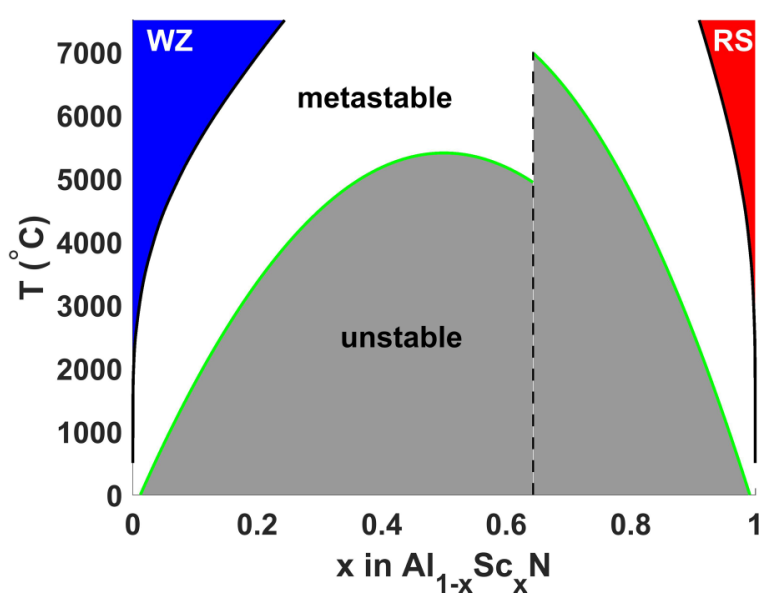

FIG. 4. Calculated temperature-composition phase diagram for $\mathrm{Al}_{1-x} \mathrm{Sc}_{x} \mathrm{~N}$ alloy with spinodal (green) and binodal (black) lines separating the regions of stability, metastability, and instability.

single-phase alloys grown in this work are achieved at thermodynamic conditions deep within the spinodal region (gray region in Fig. 4). In this region, the single-phase alloys are thermodynamically unstable towards phase separation into the end-member compounds and towards composition fluctuations that increase the composition modulation of the material (spinodal decomposition). This decomposition pathway has been experimentally observed by Höglund $e t a l$. by annealing metastable cubic $\mathrm{Al}_{0.43} \mathrm{Sc}_{0.57} \mathrm{~N}$ to enhance the decomposition kinetics. Deng et al. determined the same thing at $x=0.4$ and, when deposited with substrate temperatures greater than $400^{\circ} \mathrm{C}$, this process is observable at $x=0.3[6]$.

New perspectives from heterostructural considerations are needed to understand this behavior as the atomistic mechanisms for these two different decomposition pathways differ and, therefore, result in different kinetic barriers and metastabilities. At conditions above the binodal line, the alloy is stable and thermodynamically miscible in a single phase. Between the binodal and spinodal lines, the alloy is metastable towards composition fluctuations. The unusual behavior observed in the phase diagram of the decoupling of the binodal and spinodal miscibility gap temperatures and discontinuous spinodal line is in contrast to traditional isostructural alloy diagrams. This behavior is due to the incommensurate lattices of wurtzite and rocksalt, which require a reconstructive phase transformation at the boundary between the two phases, as demonstrated for a similar wurtzite-rocksalt heterostructure alloy $\mathrm{Mn}_{1-x} \mathrm{Zn}_{x} \mathrm{O}$ by Holder $e t a l$. and seen in the $\mathrm{Al}_{1-x} \mathrm{Sc}_{x} \mathrm{~N}$ alloy by Höglund et al. where the decomposition of metastable wurtzite with $x=0.43$ decomposed into two phases near $1100^{\circ} \mathrm{C}$.

The lattice parameter calculations shown in Fig. 5 are consistent with results of $\mathrm{x}$-ray diffraction (XRD) and SAED (see experimental results) and reported structural changes in other literature [58-60]; however, they contradict the findings of Höglund et al. [34]. Of particular note is the rapid decrease in the $c / a$ ratio of the wurtzite structure (and its approach to the hexagonal boron-nitride $c / a$ ratio) when approaching the critical composition where the rocksalt structure becomes the lowest energy. The lattice is calculated to behave with an alloying-induced expansion similar to the HBN phase below
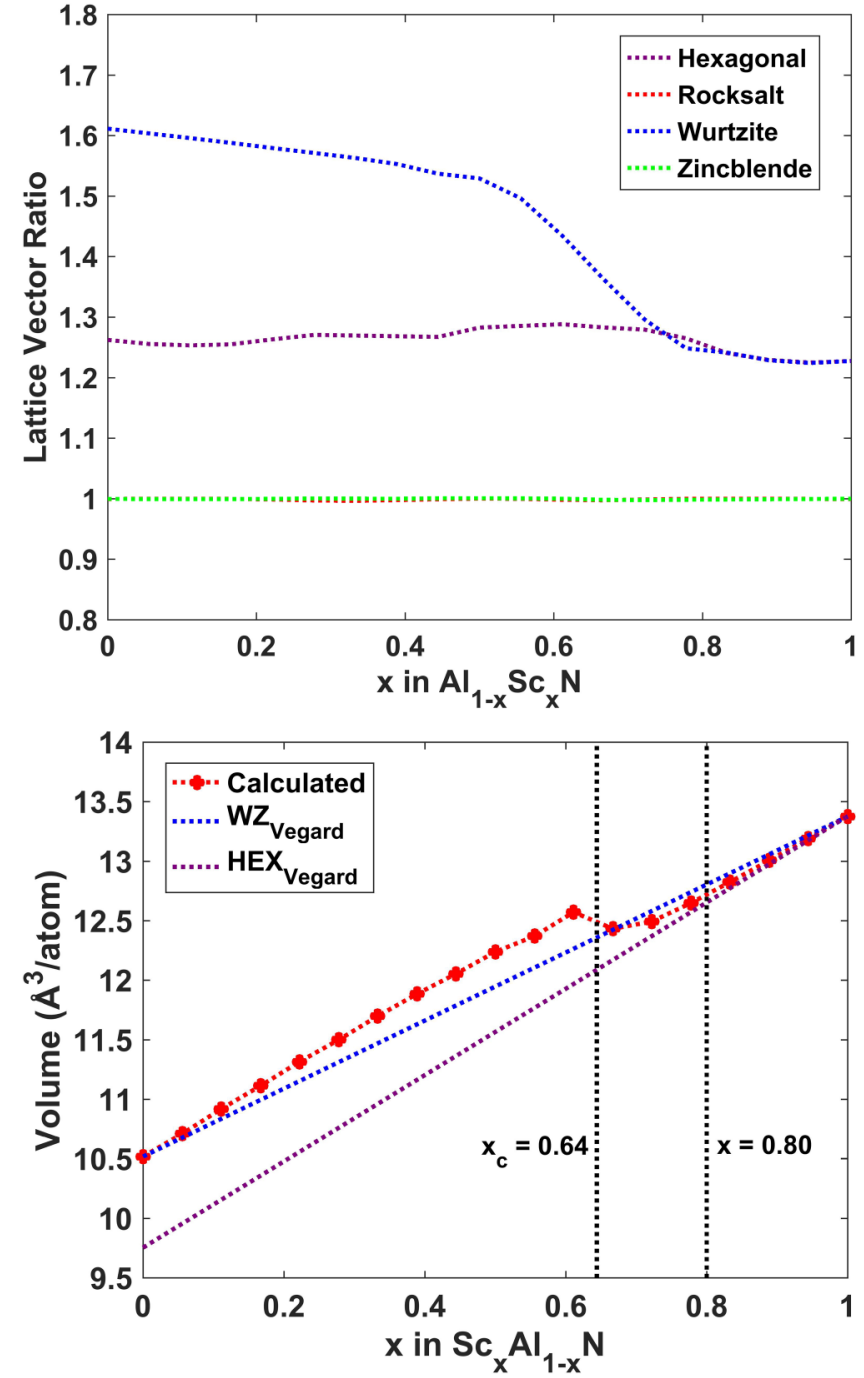

FIG. 5. Top: Calculated lattice vector ratio $c / a$ for the polymorphs considered in this study. Zinc blende and rocksalt are both unity as cubic structures. Wurtzite and hexagonal (referring to the HBN structure) are seen as compositionally dependent, where wurtzite trends towards a decreased vector ratio due to a decrease in $c$ and an increase in $a$. Bottom: Calculated volume per atom in the wurtzite and hexagonal phases compared to a linear prediction of Vegard's law [61]. In the wurtzite phase, the calculations predict a change in density which has similar behavior to the HBN Vegard relationship, resulting in a higher-volume, more-compliant wurtzite phase, finally densifying and transitioning to the hexagonal lattice in the range of $0.60<x<0.80$.

the critical composition; above $x=0.64$, the rapid densification occurs until $x=0.80$, i.e., the critical composition for transition to the HBN structure from the wurtzite phase. This structural densification occurs with increasing $x$ because the anisotropic (polar-)covalent bonding of AlN that supports the larger-volume wurtzite structure is overcome by the isotropic ionic bonding of $\mathrm{ScN}$ that facilitates the close packing of the denser HBN phase. The crossover in bond character of the alloy has important ramifications on the predicted electromechanical properties and design of piezoelectric heterostructural alloys (vide infra). The volume changes in the lattice explain why the 

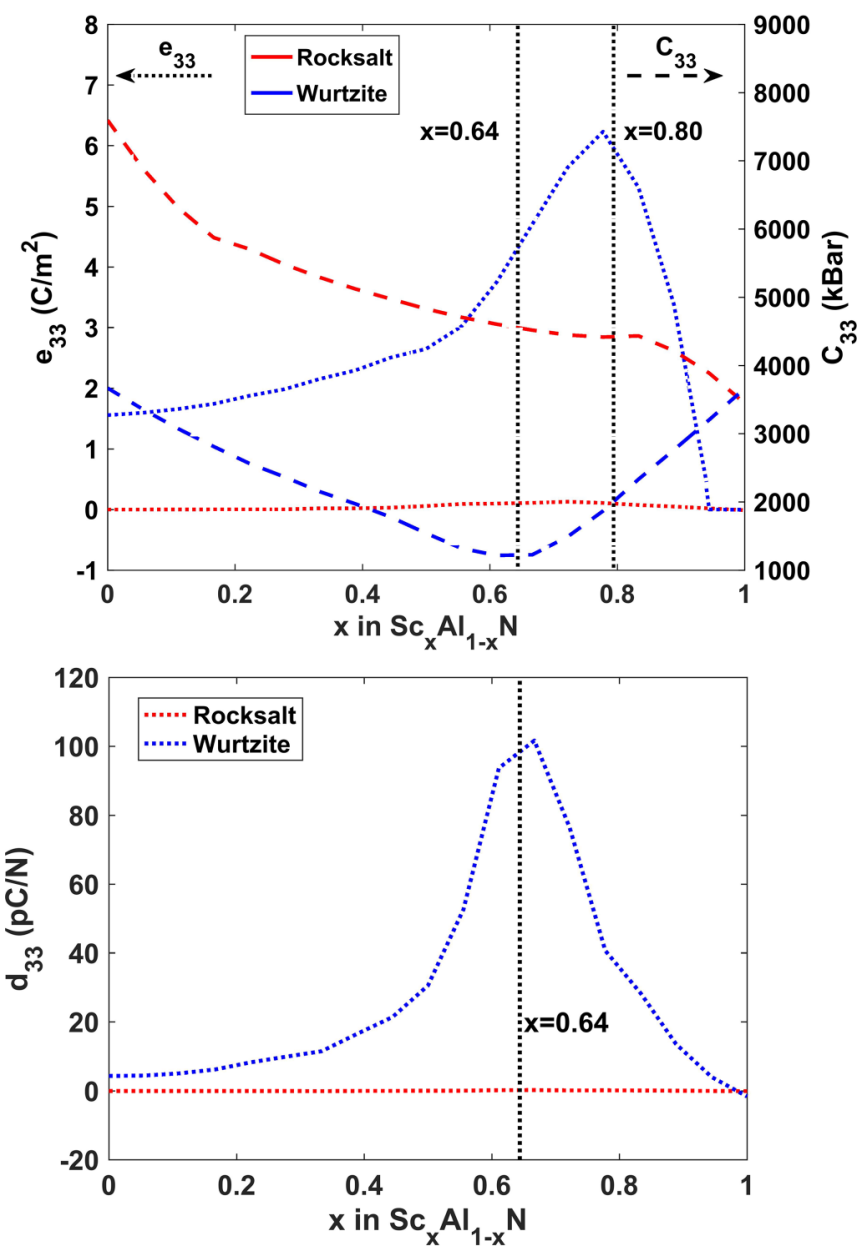

FIG. 6. Calculated (bottom) piezoelectric strain coefficient $\left(d_{33}\right)$ resulting from (top) elastic constant $\left(C_{33}\right)$ and piezoelectric stress coefficient $\left(e_{33}\right)$ for $\mathrm{Al}_{1-x} \mathrm{Sc}_{x} \mathrm{~N}$ wurtzite and rocksalt structures. The piezoelectric stress coefficient increases with increased $x$, with a maximum corresponding to the wurtzite-to-hexagonal boron-nitride critical composition. The elastic stress-strain coefficient along the polar axis has a minimum at the wurtzite-to-rocksalt critical composition. As a result, the piezoelectric strain has a maximum between the two critical compositions.

ability to achieve single-phase wurtzite alloys improves under epitaxial tensile strain, as reported by Žukauskaite $e t$ al.

The incommensurate relationship between the parent structures results in a substantial amount of lattice frustration near this phase boundary, and the (energetic) proximity of the HBN structure results in a relatively flat free-energy landscape. These two criteria (structural frustration and flat free energy) have long been seen as desired characteristics in the search for high-strain piezoelectric materials. The computationally predicted electromechanical properties for this alloy system are shown in Fig. 6 . The calculated wurtzite $d_{33}$ value increases nonlinearly from $5 \mathrm{pC} / \mathrm{N}$ at $x=0$ to $>100 \mathrm{pC} / \mathrm{N}$ above the critical composition from wurtzite to rocksalt. The structural frustration that arises from scandium substitutions in combination with the increased number of scandium-nitrogen bonds causes the lattice to soften $C$ and the piezoelectric stress coefficient $e$ to increase, resulting in the commonly observed piezoelectric strain $(d)$ coefficient increase as $d=e / C$.

\section{Rock Salt Phase Fraction}
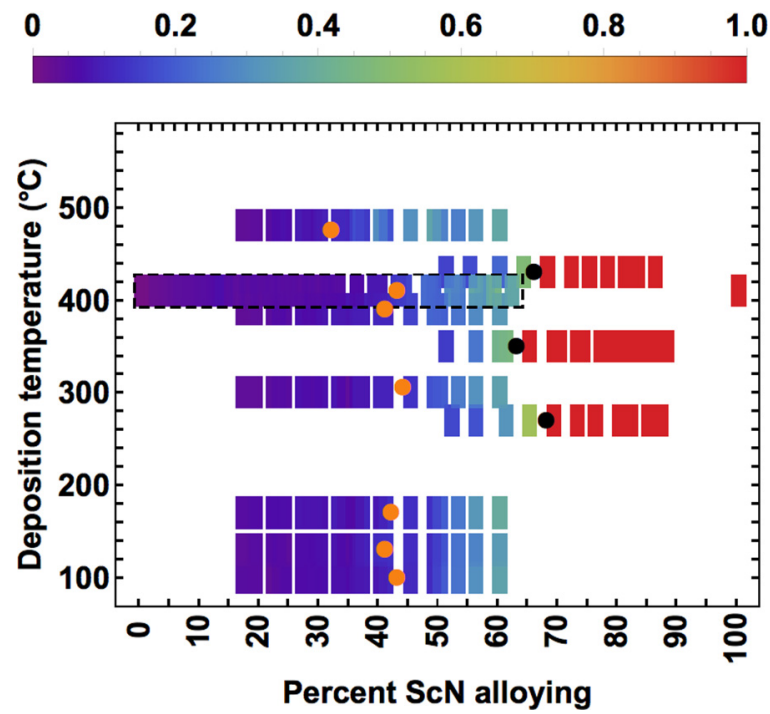

FIG. 7. Rocksalt phase fraction as a function of composition and substrate temperature during film growth. The orange points (left) are the compositions where the rocksalt phase begins to form. Similarly the black points (right) indicate the wurtzite phase-disappearance points. All samples shown are on $\mathrm{SiO}_{2}$ substrates, except the highlighted row which is on $p \operatorname{Si}(100)$. The structural trend is monotonic, suggesting that there is no observable influence from substrate effects, as expected with the amorphous surface of $\mathrm{SiO} 2$ and large lattice mismatch with crystalline silicon.

The $C_{33}$ elastic constant decreases, which is minimized near the critical composition for wurtzite to rocksalt, are attributed to the higher-volume wurtzite structure, while the piezoelectric stress constant $e_{33}$ increases with a maximum corresponding to the wurtzite-to-HBN critical composition. The results qualitatively agree with previous calculations of the elastic properties $[35,37,62,63]$ and piezoelectric properties $[35,37]$, while simultaneously extending the range of alloy space examined, which is necessary to accurately determine the decoupled nature of the responses. The results agree with the observed softening $[22,64]$ and enhanced piezoelectric strain values [31,65-67] of compositions studied in the literature. It should be noted that many methods are used in these measurements and reliable methods are just now being developed $[63,64,68]$. The predicted property behavior motivates the targeting of materials that exhibit this flattened energy landscape for enabling the decoupled tuning of the material compliance and piezoelectric properties for different target applications.

\section{B. Experiment}

Figure 7 summarizes the phase fraction analysis carried out on all sample libraries described in Table I. For substrate temperatures at or below $400^{\circ} \mathrm{C}$, films with less than roughly $40 \% \mathrm{ScN}$ content exhibit a single-phase wurtzite structure, while those with greater than roughly $65 \% \mathrm{ScN}$ exhibit a single-phase rocksalt structure, in agreement with previous studies $[4,67,69,70]$. Films of intermediate compositions contain a mixture of both wurtzite and rocksalt phases. At higher 


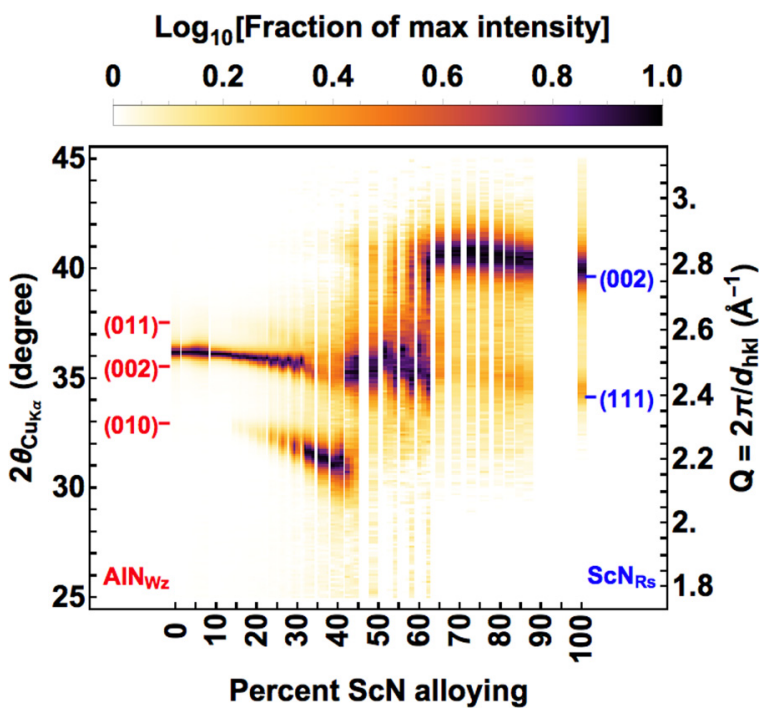

FIG. 8. Experimental x-ray diffraction profiles for a majority of the alloying space compared to the end-member peak positions of AlN [73] and $\mathrm{ScN}$ [74]. All films shown were deposited on $p \operatorname{Si}(100)$ with a substrate temperature of approximately $410^{\circ} \mathrm{C}$.

substrate temperatures, the wurtzite+rocksalt mixed-phase region expands slightly towards lower $\mathrm{ScN}$ alloying levels, suggesting that for these compositions, the single-phase wurtzite state is kinetically incapable of overcoming the barriers to phase separation and that with adequate thermal energy and diffusion time, decomposition would result in a mixture of wurtzite and rocksalt phases. This is agreement with rapid thermal annealing (RTA) experiments performed by Wu et al. but unseen for $x=0.27$ by Mayrhofer et al. in short RTA processes. This explains the early claims that these materials were thermally stable when annealed at low temperatures [5]. There is no such obvious relationship on the $\mathrm{ScN}$-rich side of the two-phase region, suggesting either that the rocksalt phase over these composition regions is more stable or that the energetic and/or kinetic barriers to decomposition are higher.

Additionally, the phase evolution of thin films deposited on $p$-type (100) oriented silicon is in agreement with that observed on amorphous $\mathrm{SiO}_{2}$ substrates, indicating that these results are not strongly dependent upon substrate choice. No changes are observed between films of different thicknesses and similar compositions, suggesting the film thickness has no impact on the phase or texture over the thickness range studied here, although the film thickness is reported elsewhere to have an effect on film stress [11], which can result in a shift in the energy landscape not accounted for within this study.

Previous studies $[6,15,71,72]$ have indicated that substrate temperatures at or near $400^{\circ} \mathrm{C}$ result in the highest film quality for reactively sputtered $\mathrm{Al}_{1-x} \mathrm{Sc}_{x} \mathrm{~N}$, so more detailed diffraction results are shown in Fig. 8 for sample libraries deposited with a substrate temperature near $400^{\circ} \mathrm{C}$. AlN with $<20 \%$ $\mathrm{ScN}$ exhibits a well-defined $(00 l)$ texture in which the material grows with the hexagonal close-packed planes parallel to the substrate, as described by Xu et al. Between approximately $20 \%$ and $40 \% \mathrm{ScN}$, the material exists in a single wurtzite phase, but grows with an increasingly polycrystalline (reducedtexture) microstructure. Above approximately $40 \% \mathrm{ScN}$, both

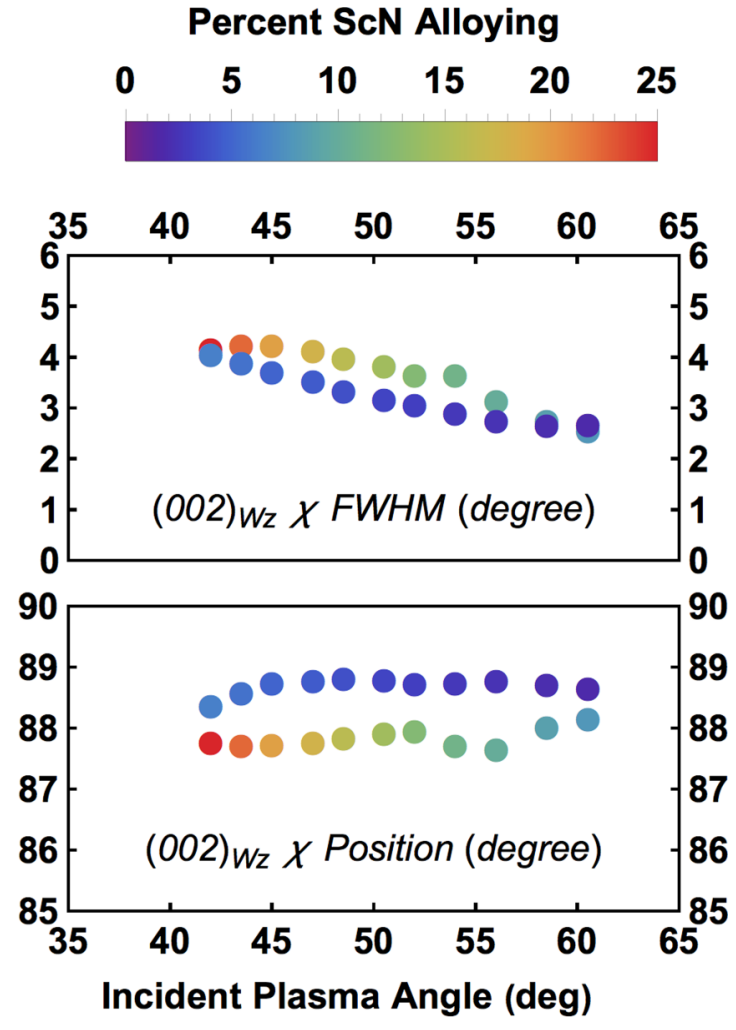

FIG. 9. Full width at half maximum and position of the wurtzite (002) peak in $\chi$ dimension, equivalent to a traditional $\omega$ rocking curve. The quality of the film texture is limited by the off-normal sputtering conditions of the combinatorial experiment seen in Fig. 2, and not by the $\mathrm{ScN}$ alloying content. The position of the peak shows a consistent tilting of the grains from the $c$-axis normal also linked to the plasma angle.

wurtzite and rocksalt phases coexist (as shown in Fig. 7), and the wurtzite phase again exhibits a strong $(00 l)$ texture. Above $65 \% \mathrm{ScN}$, the films are untextured, single-phase rocksalt. Over the entire composition range, the peak positions shift to smaller $2 \theta$ values with increased $\mathrm{ScN}$ content, indicating that the larger Sc atoms cause an expansion of the lattice as they replace smaller $\mathrm{Al}$ atoms. The shift of the wurtzite (010) peak is more dramatic than the shifting of the (002) peak, indicating a decreased $c / a$ ratio with increased $\mathrm{ScN}$ content. Experimentally observed structural changes agree with calculations of the increased $c / a$ lattice vector ratio as the structure expands in the close-packed (100) directions more than in the (001) direction. This suggests that the structure is trending towards the HBN structure depicted in Fig. 1, though no evidence of a complete phase transition to the HBN phase is observed.

For piezoelectric applications, a strong (002) texture is desired for $\mathrm{Al}_{1-x} \mathrm{Sc}_{x} \mathrm{~N}$ films. Figure 9 shows the full width at half maximum (FWHM) results from fits to the $2 \theta$ integration of the wurtzite (002) diffraction peak. The FWHM ranges from 2.5 to 4.5 degrees in $\chi$. The variation in FWHM does not appear to have a compositional dependence, but rather a dependence on the sputtering incident angle inherent to the combinatorial sputtering technique. In addition to the wurtzite (002) peak broadening quantified by this FWHM, Fig. 9 shows that the 
position of the peak deviates from 90 degrees in $\chi$, indicating that the crystallographic $c$ axis tilts increasingly away from the substrate normal with increased incident plasma angle. Thus, while the overall phase(s) and degree of texture of the films are driven by composition (and to a lesser extent, substrate temperature), the quality of the texture, as represented by the peak widths and alignment with the substrate, is more strongly related to deposition geometry.

The findings represented in Fig. 4 beg further investigation into the phase-separation process noted earlier in relation to Figs. 8 and 9. TEM was therefore employed to investigate whether the phases identified by XRD at multimicron length scales are representative of the phases present at submicron length scales and to provide insight into the film microstructure. Three compositions were chosen for investigation: $31 \%$ and $35 \% \mathrm{ScN}$ represented compositions approaching the multiphase regions but for which no rocksalt phase was observed by $\mathrm{XRD}$, and $57 \% \mathrm{ScN}$ represented the two-phase region where wurtzite and rocksalt were both well defined in XRD patterns. Note that all three of these compositions fall under the spinodal dome of Fig. 4 if considering only the substrate temperature; however, since reactive sputtering is a highly energetic and dynamic process, assigning a specific effective temperature can be misleading.

As seen in Fig. 10, at $31 \% \mathrm{ScN}$ alloying, a pure wurtzite structure is observed in selected area electron diffraction (SAED), with cell volume 6\% greater than that of AlN [74], indicating an expansion of the lattice to accommodate the scandium substitutions. At a slightly higher alloying of $35 \% \mathrm{ScN}$, the wurtzite lattice expands further to $12 \%$ larger than $\mathrm{AlN}$, and a single diffraction spot that could be attributed to the rocksalt structure is observed via SAED. The position of the rocksalt diffraction spot shows a rocksalt structure with a volume that is $6 \%$ smaller than the volume of $\mathrm{ScN}[75,76]$, suggesting aluminum incorporation in the rocksalt structure. At $57 \% \mathrm{ScN}$ alloying, the wurtzite lattice is essentially unchanged from that observed for the $35 \% \mathrm{ScN}$ composition; however, a stronger intensity is seen for the rocksalt structure.

In addition, a decrease in grain size and texture is observed across the transition region from single-phase wurtzite to single-phase rocksalt. This composition is near the transition where the wurtzite phase disappears altogether. Chemical mapping of the film cross sections using energy dispersive $\mathrm{x}$-ray spectroscopy (EDS) shows only the very early stages of chemical segregation at $31 \% \mathrm{ScN}$ alloying. Although the chemical segregation is more pronounced at $35 \% \mathrm{ScN}$, the sample appears to remain structurally homogeneous. With $57 \%$ $\mathrm{ScN}$ alloying, the chemical segregation is strongly defined and corresponds to the observed structural heterogeneity in SAED and XRD.

The mechanical properties of films prepared for this study are discussed in detail elsewhere by $\mathrm{Wu}$ et al., but it is worth noting here that the compositional region over which chemical heterogeneity is observed and which appears to represent the early stages of phase separation corresponds to the compositional region in which measured elastic values fall between the values computed via DFT for pure wurtzite and pure rocksalt phases of the respective compositions. The TEM results shown in Fig. 10 confirm that this deviation corresponds to separation into two distinct phases.
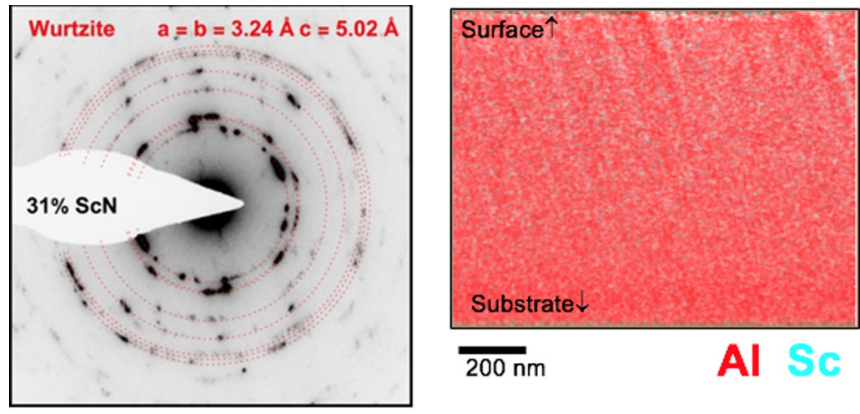

$\overline{200 \mathrm{~nm}}$

Al Sc
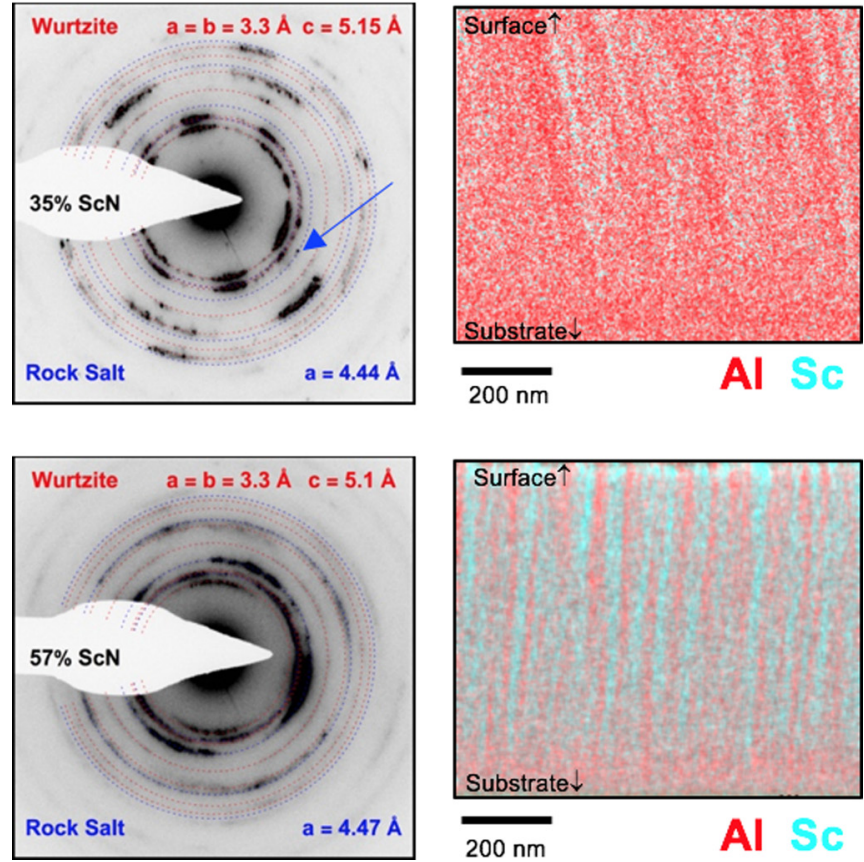

FIG. 10. Transmission electron microscopy selected area electron diffraction images (left) and corresponding $\mathrm{Al}_{1-x} \mathrm{Sc}_{x} \mathrm{~N}$ film cross sections with elemental mapping from energy-dispersive $\mathrm{x}$-ray spectroscopy (right) at three select compositions, $x=0.31,0.35$, and 0.57 (top to bottom) as determined by the global average of the elemental mapping of the film. The lowest composition $(x=0.31)$ shows only wurtzite. A small diffraction spot (blue arrow) is observed for rocksalt at $x=0.35$; however, it is primarily wurtzite. At $x=0.57$, a mix of both structures is observed.

\section{DISCUSSION}

The aluminum nitride-scandium nitride heterostructure alloy benefits from the frustration induced by these competing phases (see Fig. 5 and discussion). As the alloy incorporates larger scandium $3^{+}$ions in place of smaller $3^{+}$aluminum ions, the lattice is strained and the unit cell increases in volume. The scandium ions adopt a fourfold coordination in the wurtzite structure, increasing the enthalpy of mixing as seen in Fig. 3. The increase is found to be a minimum for the wurtzite structure with competing phases of zinc blende and hexagonal boron nitride at low and mid ScN alloying concentrations, respectively. The entropy of mixing enables a solid solution only at very low alloying concentrations or very high temperatures. More importantly, the flattening of the collective free-energy landscape leads to a broader 
thermodynamically miscible single-phase region extending further into alloy space; while not thermodynamically stable, single-phase materials can be achieved within this composition space via energetic deposition and kinetic hindrance. The energy scale of this metastability is shown to be greater than predicted previously when calculated with the optPDE van der Waals functional. It is expected that the majority of the AlN-ScN alloy system is subject to phase separation in the form of nucleation and growth at grain boundaries [34].

Large mixing enthalpies and alloy interaction parameters require highly nonequilibrium growth and subsequent kinetic hindrance to realize solid solutions, as evidenced by the highstability temperatures in the phase diagram. The difficulty of achieving high crystal quality $\mathrm{Al}_{1-x} \mathrm{Sc}_{x} \mathrm{~N}$ alloys with $x>0.2$ is explained by this, higher than previously understood, driving force for phase separation.

The combinatorial study on thin films also suggests that improved properties are seen in a region of metastability (Fig. 7), resulting in observed decomposition lines determined to be at approximately $40 \%$ and $65 \%$ for the wurtzite and rocksalt regions, respectively, at $400^{\circ} \mathrm{C}$ or less. The films exhibit compositional fluctuations and second phase formation consistent with spinodal decomposition. Combinatorial synthesis is demonstrated to be a powerful tool for validating computational insights and exploring the metastability possible through high-energy deposition processes.

\section{CONCLUSIONS}

In the case of the $\mathrm{Al}_{1-x} \mathrm{Sc}_{x} \mathrm{~N}$ alloy system, gains in performance are enabled by the energetic similarity of the HBN, rocksalt, and wurtzite phases where a single competing phase is insufficient. The kinetics required for the reconstructive phase transition required for phase separation enable synthesis of highly metastable materials and leveraging of properties affected in the process. The increased electromechanical re- sponse which spurred interest in this system reflects contributions from both an increase in the intrinsic piezoelectric coefficients and a decrease in the stiffness components. Decoupling of the piezoelectric and stiffness modifications to the influence of the HBN and rocksalt variants, respectively, offers valuable insight to the structure-property relationships in this emerging material system.

\section{ACKNOWLEDGMENTS}

K.R.T. and G.L.B. were supported by the U.S. National Science Foundation (Grant No. DMREF-1534503). J.M., S.S., B.G., A.Z., and A.M.H. were supported by the U.S. Department of Energy, Office of Science, Office of Basic Energy Sciences, as part of the Energy Frontier Research Center, Center for Next Generation of Materials by Design: Incorporating Metastability under Contract No. DE-AC3608GO28308 to the National Renewable Energy Laboratory (NREL). S.L.M. and C.B.M. acknowledge partial support from the National Science Foundation (Grant No. CBET-1433521), and S.L.M. further acknowledges support from the U.S. Department of Energy Graduate Assistance in Areas of National Need (GAANN) program (Grant No. P200A150156S). The $a b$ initio calculations were performed using High Performance Computing resources sponsored by the U.S. Department of Energy's Office of Energy Efficiency and Renewable Energy, located at NREL. The use of combinatorial thin-film synthesis and spatially resolved characterization facilities at NREL as a part of the High Throughput Experimental Materials Collaboratory (HTE-MC) is gratefully acknowledged. K.R.T. synthesized the combinatorial libraries and conducted a majority of the characterization., S.L.M., C.B.M. and A.M.H. performed the computational analysis, and S.S. and J.M. performed additional select characterization. A.M.H., A.Z., B.G., and G.L.B. provided oversight, resources, and intellectual guidance; G.L.B. conceived the overall study. All authors reviewed and contributed to the manuscript.
[1] A. Holder, S. Siol, P. Ndione, H. Peng, A. Deml, B. Matthews, L. Schelhas, M. Toney, R. Gordon, W. Tumas, J. Perkins, D. Ginley, B. Gorman, J. Tate, A. Zakutayev, and S. Lany, Sci. Adv. 3, e1700270 (2017).

[2] J. C. Schuster and J. Bauer, J. Less-Common Met. 109, 345 (1985).

[3] N. Takeuchi, Phys. Rev. B 65, 045204 (2002).

[4] C. Höglund, J. Bareño, J. Birch, B. Alling, Z. Czigány, and L. Hultman, J. Appl. Phys. 105, 113517 (2009).

[5] M. Akiyama, T. Kamohara, K. Kano, A. Teshigahara, Y. Takeuchi, and N. Kawahara, Adv. Mater. 21, 593 (2009).

[6] A. Zukauskaite, G. Wingqvist, J. Palisaitis, J. Jensen, P. Persson, R. Matloub, P. Muralt, K. Yunseok, J. Birch, and L. Hultman, J. Appl. Phys. 111, 093527 (2012).

[7] J.-c. Yang, X.-q. Meng, C.-t. Yang, and Y. Zhang, Appl. Surf. Sci. 287, 355 (2013).

[8] P. M. Mayrhofer, C. Eisenmenger-Sittner, M. Stöger-Pollach, H. Euchner, A. Bittner, and U. Schmid, J. Appl. Phys. 115, 193505 (2014).
[9] Y. Zhang, W. Zhu, D. Zhou, Y. Yang, and C. Yang, J. Mater. Sci.: Mater. Electron. 26, 2151 (2015).

[10] Y. Zhang, W. Zhu, D. Zhou, Y. Yang, and C. Yang, J. Mater. Sci.: Mater. Electron. 26, 472 (2015).

[11] S. Barth, D. Gloess, H. Bartzsch, P. Frach, T. Herzog, S. Walter, H. Heuer, G. Suchaneck, J. Juuti, J. Palosaari, and F. Ikts-md, IEEE Trans. Ultrason. Ferroelectr. Freq. Control 61, 1329 (2014).

[12] S. Fichtner, T. Reimer, S. Chemnitz, F. Lofink, and B. Wagner, APL Mater. 3, 116102 (2015).

[13] G. Wingqvist, F. Tasnádi, A. Zukauskaite, J. Birch, H. Arwin, and L. Hultman, Appl. Phys. Lett. 97, 112902 (2010).

[14] M. Akiyama, T. Tabaru, K. Nishikubo, A. Teshigahara, and K. Kano, J. Ceram. Soc. Jpn. 118, 1166 (2010).

[15] M. Akiyama, K. Kano, and A. Teshigahara, Appl. Phys. Lett. 95, 162107 (2009).

[16] R. Takei, N. Makimoto, T. Tabaru, M. Akiyama, T. Itoh, and T. Kobayashi, in 2017 19th International Conference on Solid-State 
Sensors, Actuators and Microsystems (TRANSDUCERS) (IEEE, Piscataway, NJ, 2017), pp. 1879-1882.

[17] Q. Wang, Y. Lu, S. Mishin, Y. Oshmyansky, and D. A. Horsley, J. Microelectromech. Syst. 26, 1132 (2017).

[18] M. Moreira, J. Bjurström, I. Katardjev, and V. Yantchev, Vacuum 86, 23 (2011).

[19] K. y. Hashimoto, T. Fujii, S. Sato, T. Omori, C. Ahn, A. Teshigahara, K. Kano, H. Umezawa, and S. I. Shikata, in 2012 IEEE International Ultrasonics Symposium (IEEE, Piscataway, NJ, 2012), pp. 1-4.

[20] M. A. Moreira, J. Bjurström, V. Yantchev, and I. Katardjiev, IOP Conf. Ser.: Mater. Sci. Eng. 41, 012014 (2012).

[21] K. Umeda, H. Kawai, A. Honda, M. Akiyama, T. Kato, and T. Fukura, in 2013 IEEE 26th International Conference on Micro Electro Mechanical Systems (MEMS) (IEEE, Piscataway, NJ, 2013), pp. 733-736.

[22] R. Matloub, A. Artieda, C. Sandu, E. Milyutin, and P. Muralt, Appl. Phys. Lett. 99, 092903 (2011).

[23] M. Schneider, M. DeMiguel-Ramos, A. J. Flewitt, E. Iborra, and U. Schmid, Proceedings 1, 305 (2017).

[24] B. A. Griffin, M. D. Henry, R. W. Reger, and B. Heinz, in 2017 IEEE International Ultrasonics Symposium (IUS) (IEEE, Piscataway, NJ, 2017), pp. 1-4.

[25] L. Colombo, A. Kochhar, C. Xu, G. Piazza, S. Mishin, and Y. Oshmyansky, in Investigation of $20 \%$ Scandium-Doped Aluminum Nitride Films for MEMS Laterally Vibrating Resonators (IEEE, Piscataway, NJ, 2017).

[26] M. Suzuki, T. Yanagitani, and H. Odagawa, in Polarization Inverted (0001)/(000-1) ScAIN Film Resonators Operating in Second Overtone Mode (IEEE, Piscataway, NJ, 2012).

[27] A. Konno, M. Sumisaka, A. Teshigahara, K. Kano, K.-y. Hashimo, H. Hirano, M. Esashi, M. Kadota, and S. Tanaka, in ScAlN Lamb Wave Resonator in $\mathrm{GHz}$ Range Released by XeF2 Etching (IEEE, Piscataway, NJ, 2013).

[28] V. Pashchenko, S. Mertin, F. Parsapour, J. Li, P. Muralt, and S. Ballandras, in 2017 Joint Conference of the European Frequency and Time Forum and IEEE International Frequency Control Symposium (EFTF/IFC) (IEEE, Piscataway, NJ, 2017).

[29] S. Mishin, M. Gutkin, A. Bizyukov, and V. Sleptsov, in Method of Controlling Coupling Coefficient of Aluminum Scandium Nitride Deposition in High Volume Production (IEEE, Piscataway, NJ, 2013), pp. 126-128.

[30] S. Mishin and Y. Oshmyansky, in Manufacturability of Highly Doped Aluminum Nitride Films (IEEE, Piscataway, NJ, 2015).

[31] S. Fichtner, N. Wolff, G. Krishnamurthy, A. Petraru, S. Bohse, F. Lofink, S. Chemnitz, H. Kohlstedt, L. Kienle, and B. Wagner, J. Appl. Phys. 122, 035301 (2017).

[32] Y. Lu, M. Reusch, N. Kurz, A. Ding, T. Christoph, L. Kirste, V. Lebedev, and A. Žukauskaitè, Phys. Status Solidi A 1700559 (2017).

[33] C. Höglund, J. Birch, B. Alling, J. Bareño, Z. Czigány, P. O. Persson, G. Wingqvist, A. Zukauskaite, and L. Hultman, J. Appl. Phys. 107, 123515 (2010).

[34] C. Höglund, B. Alling, J. Birch, M. Beckers, P. O. A. Persson, C. Baehtz, Z. Czigány, J. Jensen, and L. Hultman, Phys. Rev. B 81, 224101 (2010).

[35] F. Tasnadi, B. Alling, C. Hoglund, G. Wingqvist, J. Birch, L. Hultman, and I. A. Abrikosov, Phys. Rev. Lett. 104, 137601 (2010).
[36] H. Berkok, A. Tebboune, A. Saim, and A. H. Belbachir, Physica B: Phys. Condens. Matter 411, 1 (2012).

[37] M. A. Caro, S. Zhang, T. Riekkinen, M. Ylilammi, M. A. Moram, O. Lopez-Acevedo, J. Molarius, and T. Laurila, J. Phys.: Condens. Matter 27, 245901 (2015).

[38] P. Daoust, P. Desjardins, R. A. Masut, V. Gosselin, and M. Côté, Phys. Rev. Mater. 1, 055402 (2017).

[39] E. Iborra, J. Capilla, J. Olivares, M. Clement, and V. Felmetsger, in Piezoelectric and Electroacoustic Properties of Ti-doped AlN thin Films as a Function of Ti Content (IEEE, Piscataway, NJ, 2012).

[40] H. Liu, F. Zeng, G. Tang, and F. Pan, Appl. Surf. Sci. 270, 225 (2013).

[41] A. Kabulski, V. Pagan, and D. Korakakis, Mater. Res. Soc. Symp. Proc. 1129 (2009).

[42] V. Felmetsger and M. Mikhov, in Reactive Magnetron Sputtering of Piezoelectric Cr-Doped AlN Thin Films (IEEE, Piscataway, NJ, 2011).

[43] A. Zakutayev, T. R. Paudel, P. F. Ndione, J. D. Perkins, S. Lany, A. Zunger, and D. S. Ginley, Phys. Rev. B 85, 085204 (2012).

[44] S. Siol, P. Schulz, M. Young, K. A. Borup, G. Teeter, and A. Zakutayeve, Adv. Mater. Interf. 3, 1600755 (2016).

[45] S. Siol, A. Holder, B. O. Ortiz, P. A. Parilla, E. Toberer, S. Lany, and A. Zakutayev, RSC Adv. 7, 24747 (2017).

[46] H. Peng, P. F. Ndione, D. S. Ginley, A. Zakutayev, and S. Lany, Phys. Rev. X 5, 021016 (2015).

[47] A. Zunger, S. H. Wei, L. G. Ferreira, and J. E. Bernard, Phys. Rev. Lett. 65, 353 (1990).

[48] G. Kresse and J. Furthmuller, Phys. Rev. B 54, 11169 (1996).

[49] G. Kresse and D. Joubert, Phys. Rev. B 59, 1758 (1999).

[50] J. P. Perdew, K. Burke, and M. Ernzerhof, Phys. Rev. Lett. 77, 3865 (1996).

[51] S. Grimme, J. Comput. Chem. 27, 1787 (2006).

[52] J. Harl and G. Kresse, Phys. Rev. B 77, 045136 (2008).

[53] J. Klimeš, D. R. Bowler, and A. Michaelides, J. Phys.: Condens. Matter 22, 022201 (2009).

[54] S. Baroni, S. de Gironcoli, A. Dal Corso, and P. Giannozzi, Rev. Mod. Phys. 73, 515 (2001).

[55] A. Bikowski, S. Siol, J. Gu, A. Holder, J. Mangum, B. Gorman, W. Tumas, S. Lany, and A. Zakutayev, Chem. Mater. 29, 6511 (2017).

[56] See Supplemental Material at http://link.aps.org/supplemental/ 10.1103/PhysRevMaterials.2.063802 for further details on computational methods and phase analysis.

[57] L. A. Giannuzzi and F. A. Stevie, Micron 30, 197 (1999).

[58] S. Zhang, D. Holec, W. Y. Fu, C. J. Humphreys, and M. A. Moram, J. Appl. Phys. 114, 133510 (2013).

[59] R. Deng, S. R. Evans, and D. Gall, Appl. Phys. Lett. 102, 112103 (2013).

[60] A. Žukauskaitè, C. Tholander, F. Tasnádi, B. R. Alling, J. Palisaitis, J. Lu, P. O. Å. Persson, L. Hultman, and J. Birch, Acta Mater. 94, 101 (2015).

[61] L. Vegard, Z. Phys. 5, 17 (1921).

[62] S. Zhang, W. Y. Fu, D. Holec, C. J. Humphreys, and M. A. Moram, J. Appl. Phys. 114, 243516 (2013).

[63] P. M. Mayrhofer, H. Euchner, A. Bittner, and U. Schmid, Sensors Actuators A (Phys.) 222, 301 (2015). 
[64] D. Wu, K. Talley, S. Manna, A. Zakutayev, C. Ciobanu, P. Constantine, G. Brennecka, and C. Packard (unpublished).

[65] A. Žukauskaite, E. Broitman, P. Sandström, L. Hultman, and J. Birch, Phys. Status Solidi A 212, 666 (2015).

[66] S. Barth, H. Bartzsch, D. Glöß, P. Frach, T. Modes, O. Zywitzki, G. Suchaneck, and G. Gerlach, Microsyst. Technol. 22, 1613 (2016).

[67] O. Zywitzki, T. Modes, S. Barth, H. Bartzsch, and P. Frach, Surf. Coat. Technol. 309, 417 (2017).

[68] P. M. Mayrhofer, E. Wistrela, M. Schneider, A. Bittner, and U. Schmid, Proc. Eng. 168, 876 (2016).

[69] R. Deng, P. Y. Zheng, and D. Gall, J. Appl. Phys. 118, 015706 (2015).
[70] W. J. Liauh, S. Wu, J.-L. Huang, D.-F. Lii, Z.-X. Lin, and W.-K. Yeh, Surf. Coat. Technol. 43rd Intl. Metallurg. Coat. Thin Films, 308, 101 (2016).

[71] S. Wu, M. Y. Wu, J.-L. Huang, and D.-F. Lii, Int. J. Appl. Ceram. Technol. 11, 894 (2014).

[72] P. M. Mayrhofer, P. O. Å. Persson, A. Bittner, and U. Schmid, Microsyst. Technol. 22, 1679 (2016).

[73] R. Wyckoff, Crystal Structures, Crystal Structures No. 1 (Wiley, New York, 1963).

[74] M. Durandurdu, J. Alloys Compd. 480, 917 (2009).

[75] X.-H. Xu, H.-S. Wu, C.-J. Zhang, and Z.-H. Jin, Thin Solid Films 388, 62 (2001).

[76] Z. T. Y. Liu, X.Zhou, S. V. Khare, and D. Gall, J. Phys.: Condens. Matter 26, 025404 (2014). 